\title{
Variable Heat Disturbance Observer for Control of Peltier Device
}

\author{
Yukiko Osawa* Member, Seiichiro Katsura* Senior Member
}

(Manuscript received April 23, 2018, revised Oct. 30, 2018)

\begin{abstract}
A thermoelectric device called a Peltier device is widely used in many fields. For example, the device is used for a cooling system such as in systems consisting of medical reagent coolants and refrigerators. However, controlling the device is difficult because thermal characteristics of the device change depending on its temperature. Notably, the amount of heat flow generated from the device varies with the amount of current applied. Therefore, a thermal nonlinear behavior has to be considered in a thermal model. Most studies do not consider the nonlinear characteristics and treat the device as a linear system. Therefore, robustness against disturbance is not high because of modeling error. This paper presents a heat disturbance observer considering the nonlinear characteristics of a Peltier device. Variable parameters depending on the temperature and current of the device are used, and a variable heat disturbance observer is proposed. The thermal model used in the proposed observer includes a few nonlinear elements such as Joule heat and heat flow inside the device. The objective of this study is to improve the response of a Peltier device. Some experiments were conducted to verify the validity of the proposed method. Besides, the effect of modeling error on the stability of the observer is discussed.
\end{abstract}

Keywords: peltier device, thermoelectric conversion elements, variable heat disturbance observer, robust temperature control

\section{Intoroduction}

A Peltier device is a thermoelectric element shown in Fig. 1, and it is widely used in many fields. For example, the device is used for a cooling system such as in systems consisting of medical reagent coolants and refrigerators. In this study, the device is used as a control object, that the output is temperature monitored from thermocouple attached to the device and the input is current. It is useful because generated heat from the device called a Peltier effect can be controlled by a current and the device can be both heat source and coolant.

The method for controlling a Peltier device has been studied in many years. As a first step, temperature control of the device has been researched ${ }^{(1)(2)}$. In addition, the control method of considering human interaction has also been studied $^{(3)}$. Temperature control technique using a quality of a material was also proposed ${ }^{(4)}$. A heat disturbance observer (HDOB) was proposed and it is possible to conduct robust temperature control against a disturbance ${ }^{(5)}$. Furthermore, the method of heat flow control has been studied ${ }^{(6)}$. A heat inflow observer (HIOB) for estimating a heat flow from outside of the device was also proposed ${ }^{(7)}$. Besides, both temperature and heat flow can be controlled using some control methods ${ }^{(8)(9)}$. By using the relationship between temperature and heat flow, the method that temperature responses are changed depending on an external heat flow has been proposed ${ }^{(10)}$.

There are some studies about a human sensation analysis focused on evaluating a thermal sensation using multiple Peltier devices ${ }^{(11)(12)}$. Human spatial and temporal resolution of the thermal sensations are changed depending on

\footnotetext{
${ }^{*}$ Department of System Design Engineering, Keio University 3-14-1, Hiyoshi, Kohoku-ku, Yokohama 223-8522, Japan
}

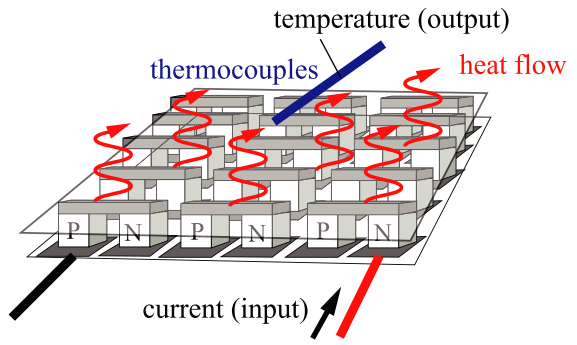

Fig. 1. Structure of a Peltier device

the situation. For example, smaller temperature differences can be detected by extending an area of rendering the thermal sensation ${ }^{(13)}$. The literature ${ }^{(14)}$ analyzed a relationship between a heated space and time duration of imposing the heat sources. Other studies ${ }^{(15)}$ also analyzed a human body perception of coolness at changing temperatures from multiple dynamically located heat sources. Some methods were developed to deliver spatial and thermal sensation based on a phantom sensation using multiple heat sources ${ }^{(16)(17)}$.

As for health care system, a Peltier device is not only utilized for controlling but also sensing. The literature ${ }^{(18)}$ developed an interface which is mounted some multiple sensors such as glucose, lactate and temperature sensors. The flexible thermal devices for health monitoring were developed that can obtain an information of a physical condition ${ }^{(19)(20)}$. Furthermore, the flexible thermoelectric element used as a temperature sensor for multiple measurements was developed ${ }^{(21)}$.

A Peltier device is composed of a metal and semiconductor, and there are three kinds of thermal resistances that a phonon boundary resistance (semiconductor), an electric boundary resistance (metal), and mixed boundary resistance (between semiconductor and metal). These resistances change depending on temperature or potential difference of 


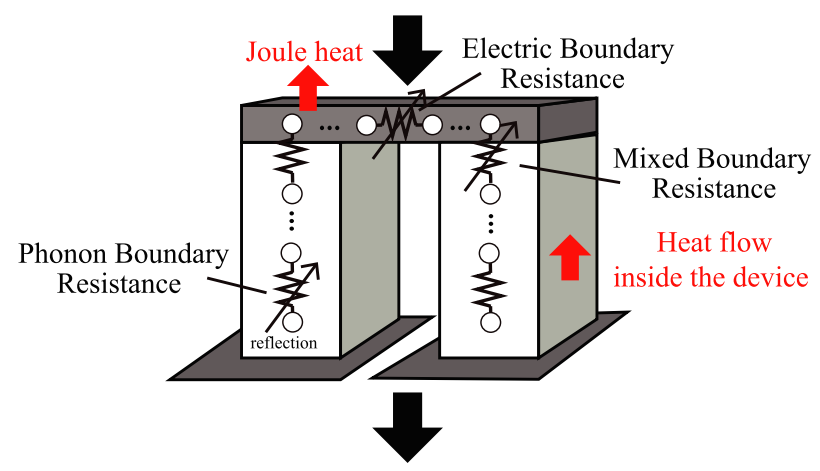

Fig. 2. Nonlinearity of a Peltier device

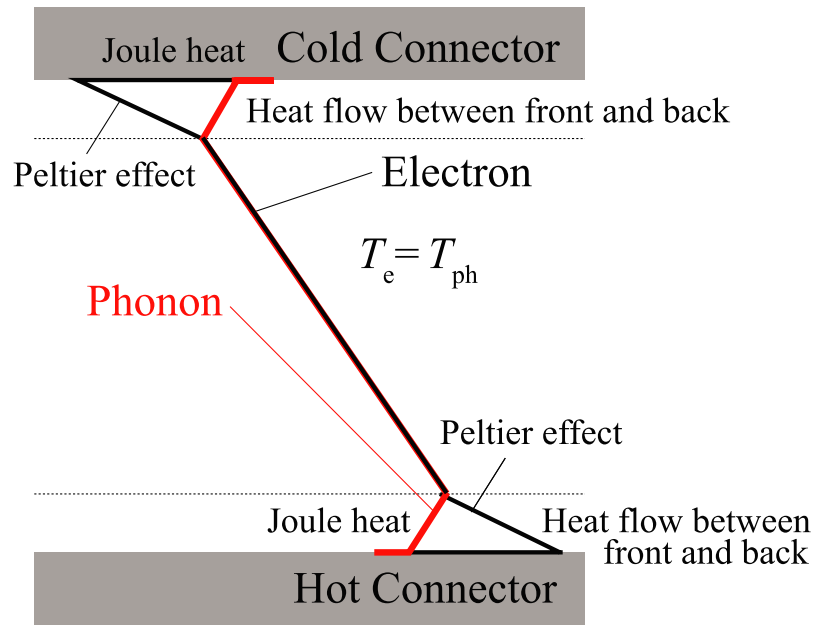

Fig. 3. Nonlinearity characteristics of transition energy

the device, and a Peltier device has a nonlinear characteristics ${ }^{(22)-(24)}$.

However, most of studies use the device as a linear system. In other words, these methods do not consider that an amount of heat flow generated from the device with the amount of current applied. There are some conventional method that a heat flow generated from the device is treated as a quadratic equation of temperature ${ }^{(25)-(27)}$. The model of these methods are close to an actual device, however, change of characteristics has to be considered.

This paper presents a heat disturbance observer considering the temperature characteristics of the Peltier device. Variable parameters depending on temperature and current of the device is used, and a variable heat disturbance observer (VHDOB) is proposed. The thermal model used in the proposed observer includes a few nonlinear elements such as a Joule heat and heat flow inside the device. The objective of this study is to improve a robustness against a disturbance of the control system. Some experiments were conducted to verify the validity of the proposed method. In addition, a stability analysis of the observer is conducted.

\section{Objective of the Proposed Method}

The objective of this study is to improve robustness against a disturbance of the control system. The disturbance treated in this paper is modeling error including the parameter variation depending on a temperature change. The modeling error becomes large when the thermal parameter changes, and it sometimes has a negative influence to control system. It

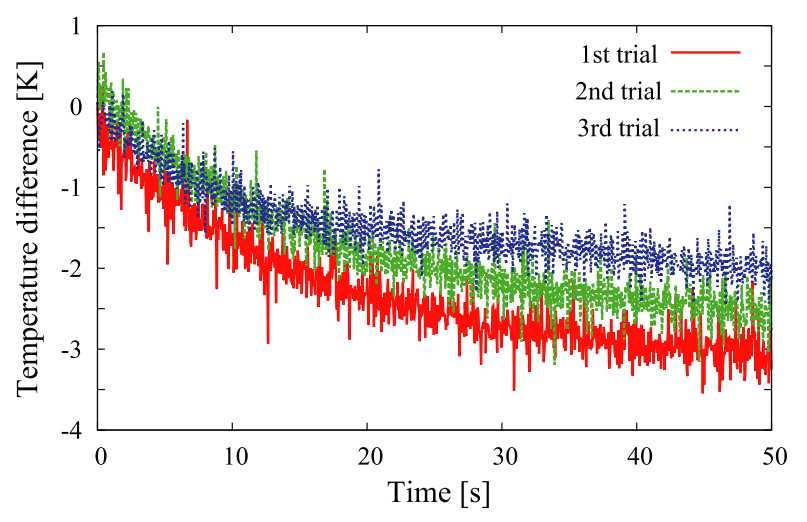

Fig. 4. Experimental results of current control

cannot be measured because the modeling error is not known. In response to this, the observer is used in this paper. The observer is defined as an algorithm for rejecting disturbance and compensating. Therefore, the control plant can be treated as a thermal model by using the disturbance. There is an HDOB that estimates a disturbance heat flow. In the conventional observer, disturbance includes modeling error of nonlinear elements such as a Joule heat. However, such a nonlinear element becomes dominant in case that heat cannot be radiated enough. The large modeling error delays the response speed of the device, and the proposed method focused on decreasing modeling error by considering nonlinear characteristics of the control target. Besides, it is limited to set a cut-off frequency of the observer because of the modeling error in the conventional one. In this paper, nonlinear elements such as a Joule heat and heat flow inside the device are considered in the model to decrease the modeling error from the nonlinear thermal behavior. By using the proposed method, it is expected to extend a field that the device can be used.

\section{Modeling of the Thermal System}

3.1 Nonlinear Elements of a Peltier Device The thermal phenomena of the Peltier device consists of some elements such as metal and semiconductor. Figure 2 shows three kinds of thermal resistances involved in the device; a phonon boundary resistance, an electric boundary resistance, and mixed boundary resistance, respectively. In each resistance, heat is generated by applying current. These resistances are changed depending on the temperature of the device and the Peltier device has a nonlinear characteristic. Figure 3 shows the transition energy of the device ${ }^{(24)}$. From Fig. 3, heat conduction of electron and phonon is correspond with each other in the middle of the device. On the other hand, Peltier effect in the phonon side and Joule heat in the electron side is independently each other and these phenomena are occurred in the different way. Therefore, the heat phenomena in the Peltier device consists of some elements and it has a nonlinear characteristic. Here, $T_{\mathrm{e}}$ and $T_{\mathrm{ph}}$ stand for the temperature of electron and phonon in the Peltier device, respectively. Figure 4 shows the experimental results of temperature responses of the device in case of applying current 0.5 A. From Fig. 4, temperature rises as the number of trial times increasing. It is because the Joule heat and heat flow between both sides of the device are generated and accumulated inside the device. Therefore, the nonlinearity has 


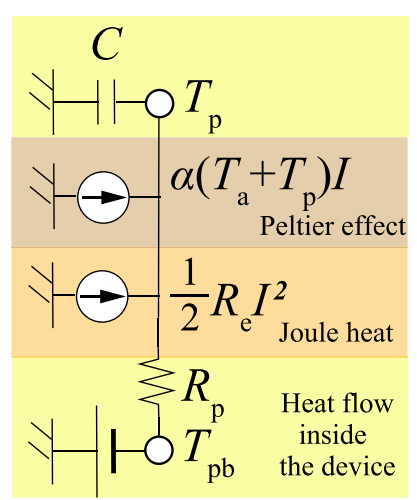

Fig. 5. Modeling of a Peltier device based on a thermal network method

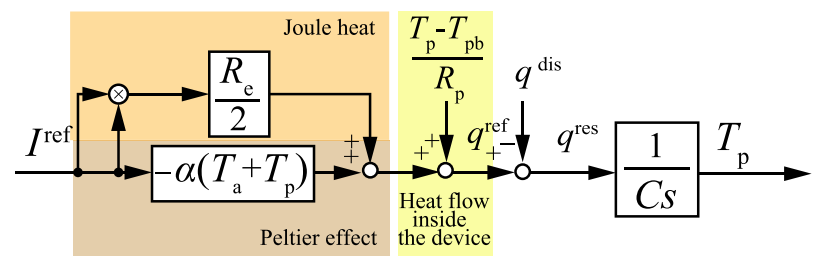

Fig. 6. Block diagram of the model of a Peltier device

to be considered in the thermal model.

3.2 Definition of Disturbance Heat Flow Most of studies use a Peltier device as a linear system; however, it has a nonlinear characteristics that an amount of the heat flow is changed depending on its temperature and its input current. The heat flow generated from a Peltier device includes heat from a Peltier effect, a Joule heat, and heat interference inside the device. The thermal system is modeled based on a thermal network method ${ }^{(28)(29)}$ that a thermal behavior is expressed as a electric circuit. In this paper, the heat flow generated from a Peltier device is expressed as

$$
q=-\alpha\left(T_{\mathrm{a}}+T_{\mathrm{p}}\right) I+\frac{1}{2} R_{\mathrm{e}} I^{2}+\frac{1}{R_{\mathrm{p}}}\left(T_{\mathrm{p}}-T_{\mathrm{pb}}\right), \cdots
$$

where $q, \alpha, T_{\mathrm{a}}, I, R_{\mathrm{e}}, R_{\mathrm{p}}, T_{\mathrm{p}}$, and $T_{\mathrm{pb}}$ stand for heat flow, Seebeck coefficient, absolute temperature, current, electric resistance, thermal resistance of a Peltier device, temperature of the front and back side of Peltier device, respectively. Equation (1) shows a general model of the Peltier device. The first term of (1) means heat generated from a Peltier effect, the second term means a Joule heat, and the third term stands for heat interference inside the device, respectively. In general, heat flow is integral value of temperature. Therefore, the temperature of the device in the Laplace domain is described based on (1) as

$$
T=\frac{\left(-\alpha\left(T_{\mathrm{a}}+T_{\mathrm{p}}\right) I+\frac{1}{2} R_{\mathrm{e}} I^{2}+\frac{1}{R_{\mathrm{p}}}\left(T_{\mathrm{p}}-T_{\mathrm{pb}}\right)\right)}{C s} .
$$

From (2), it can be said that the thermal system has a nonlinear elements in the Laplace domain. It can also be confirmed from experimental results. From some preliminary experiments, the attainment temperature is increased in proportion to the amount of the current, and the responses are changed gradually because of a Joule heat and heat flow inside the device. Thus, the model are used in this paper. The thermal system based on a thermal network method is shown in

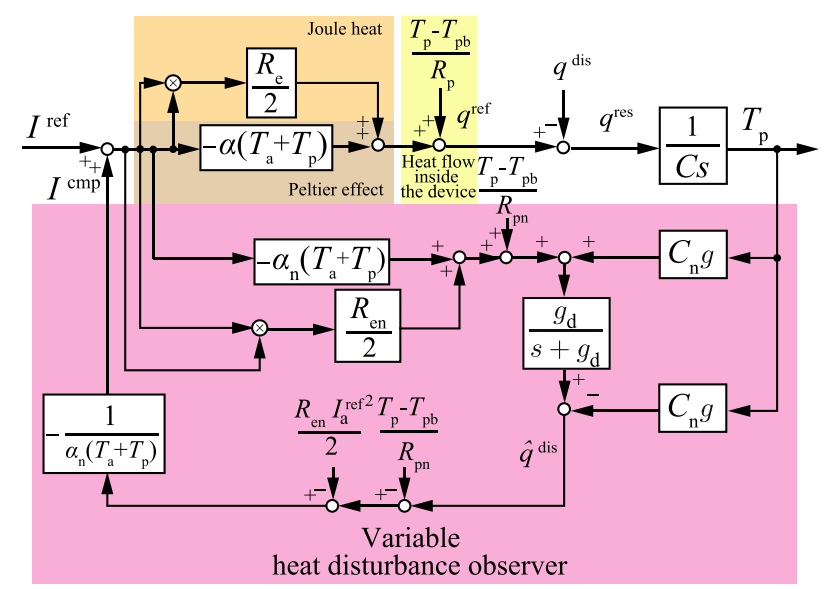

Fig. 7. Block diagram of the variable heat disturbance observer

Fig. 5. Here, a disturbance heat flow $q^{\text {dis }}$ is defined as

$$
q^{\mathrm{dis}}=\frac{1}{2} \Delta R_{\mathrm{e}} I^{2}+\frac{1}{\Delta R_{\mathrm{p}}}\left(T_{\mathrm{p}}-T_{\mathrm{pb}}\right)+\Delta \alpha T_{\mathrm{a}} I^{\mathrm{ref}}+\Delta C s T_{\mathrm{p}}
$$

where superscript dis, $\Delta$, and $C$ stand for disturbance, modeling error, and thermal capacitance of Peltier device, respectively. In the conventional method, Joule heat and heat flow inside the Peltier device is included to the disturbance heat flow $q^{\text {dis }}$, because the nonlinear elements of the device are not considered. This means that the modeling error becomes large depending on the temperature and current change. Therefore, the disturbance heat flow includes only modeling error in the proposed method.

\subsection{Modeling Peltier Device Considering Nonlinear} Characteristics As mentioned previously, a Peltier device is treated as a linear system in many conventional studies. However, the thermal characteristics is changed depending on the temperature and current of a Peltier device. From (1), there are nonlinear elements such as Joule heat and heat flow inside the device. In the proposed method, the nonlinear elements are included in the model, shown as

$$
q^{\mathrm{ref}}=-\alpha_{\mathrm{n}}\left(T_{\mathrm{a}}+T_{\mathrm{p}}\right) I^{\mathrm{ref}}+\frac{1}{2} R_{\mathrm{en}} I^{\mathrm{ref} 2}+\frac{1}{R_{\mathrm{pn}}}\left(T_{\mathrm{p}}-T_{\mathrm{pb}}\right),
$$

where superscript ref and subscript $\mathrm{n}$ stand for the reference value and the nominal value, respectively. The model is made based on a general model shown in (1), and it is used for observer.

The nonlinear model was verified by comparing to some experimental results by applying various currents. The response value of heat flow can be described as

$$
\begin{aligned}
& q^{\mathrm{res}}=-\alpha_{\mathrm{n}}\left(T_{\mathrm{a}}+T_{\mathrm{p}}\right) I^{\mathrm{ref}}+\frac{1}{2} R_{\mathrm{en}} I^{\mathrm{ref} 2}+\frac{1}{R_{\mathrm{pn}}}\left(T_{\mathrm{p}}-T_{\mathrm{pb}}\right)-q^{\mathrm{dis}} \\
& =q^{\mathrm{ref}}-q^{\mathrm{dis}} \text {, }
\end{aligned}
$$

where superscript res stands for the response value. The block diagram of a Peltier device is shown in Fig. 6. As for Joule heat, the electric resistance $R_{\mathrm{e}}$ is measured and the Joule heat can be calculated from $R_{\mathrm{e}}$ and current input. As for 


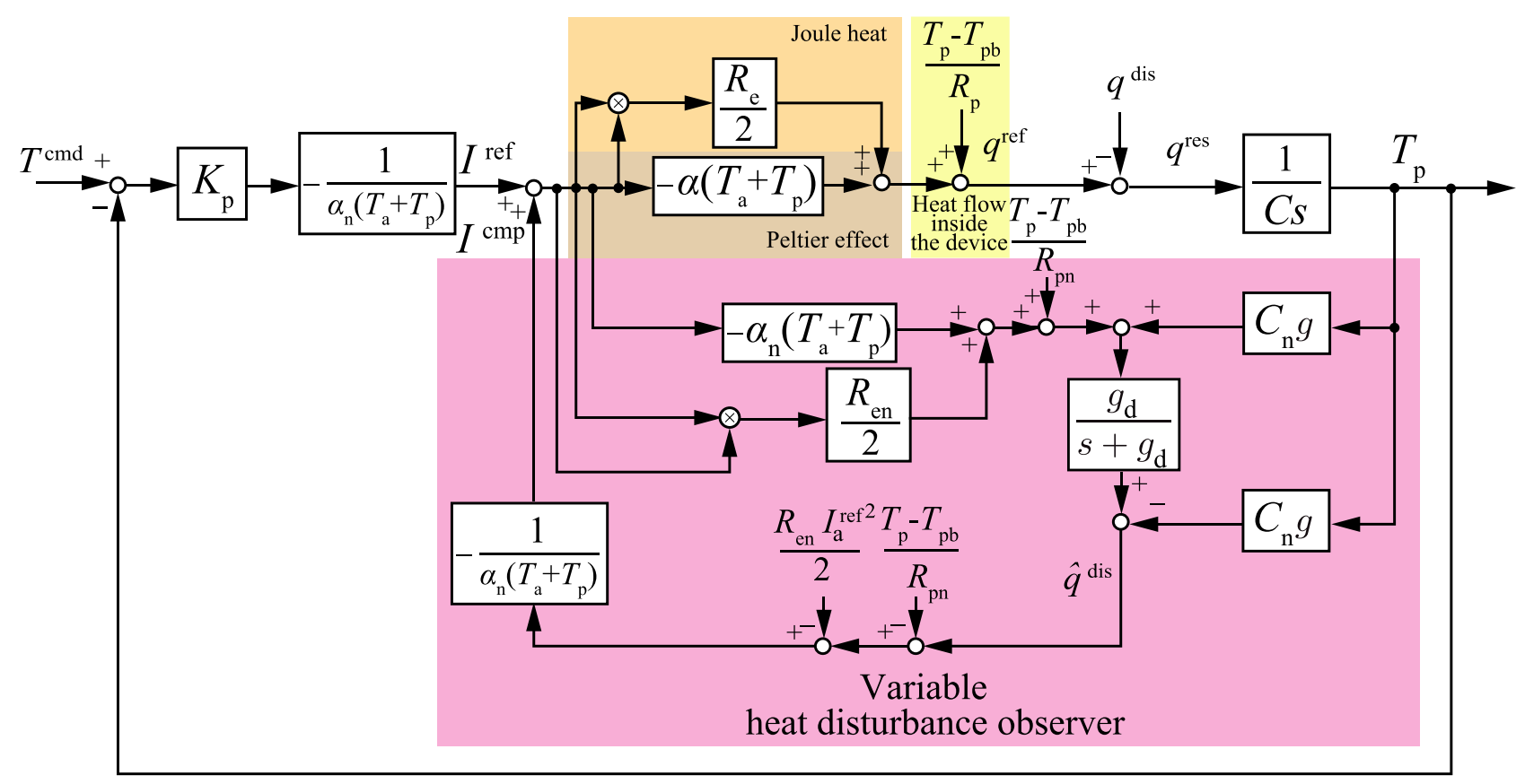

Fig. 8. Block diagram of control system of the proposed method

heat flow inside the device, the thermal resistance $R_{\mathrm{p}}$ is identified from some preliminary experiments, and temperature at both sides of the device is obtained from two thermocouples. From (4), the model of the device is changed nonlinearly depending on the temperature of the Peltier device $T_{\mathrm{p}}$ and current input $I^{\text {ref }}$. In the next section, the control system is explained based on the thermal model.

\section{Control system of a Peltier Device}

4.1 Variable Heat Disturbance Observer In the case of temperature control, there is a disturbance heat flow such as radiation of heat, Joule heat, external heat, etc. Therefore, such disturbance has to be rejected to conduct robust control. HDOB ${ }^{(5)}$ is an observer which is derived by applying a disturbance observer ${ }^{(30)}$ for the field of thermal control, and it estimates the disturbance heat flow by comparing a nominal input of heat flow with output of heat flow. It is able to estimate the disturbance heat flow by using the observer, however, response speed against disturbance becomes slow because of modeling error. In this paper, the model of a Peltier device is changed depending on its temperature and its reference value of a current to decrease the modeling error from the nonlinear thermal behavior. By using (4), the disturbance heat flow can be calculated as

$$
\hat{q}^{\text {dis }}=\frac{g_{\mathrm{d}}}{s+g_{\mathrm{d}}} q^{\mathrm{dis}} \ldots \ldots \ldots \ldots \ldots \ldots \ldots \ldots \ldots \ldots \ldots \ldots \ldots \ldots \ldots
$$

The observer proposed in this paper is defined as Variable Heat Disturbance Observer (VHDOB). The compensated current from VHDOB is calculated as

$$
I^{\mathrm{cmp}}=-\frac{1}{\alpha_{\mathrm{n}}\left(T_{\mathrm{a}}+T_{\mathrm{p}}\right)}\left(\hat{q}^{\mathrm{dis}}-\frac{1}{2} R_{\mathrm{e}} I^{2}-\frac{1}{R_{\mathrm{p}}}\left(T_{\mathrm{p}}-T_{\mathrm{pb}}\right)\right),
$$

where superscript cmp denotes command value. The block diagram of the variable heat disturbance observer is shown in

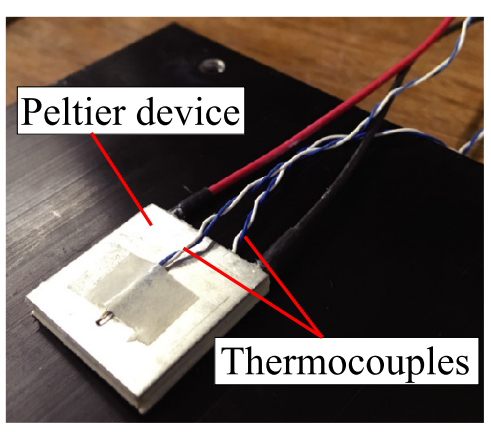

Fig. 9. Experimental setup

Fig. 7. In Fig. 7, $I_{\mathrm{a}}^{\mathrm{ref}}$ and $g_{\mathrm{d}}$ stand for current after compensation and cut-off frequency of a low-pass filter for VHDOB, respectively. In order to get temperature information at the both sides of a Peltier device, two thermocouples are used in the proposed method. By using this observer, robust temperature control can be conducted against the disturbance of heat flow. In the next section, whole control system of the proposed method is explained.

4.2 Whole Control System The block diagram of the whole control system is shown in Fig. 8. Temperature control is conducted using proportional controller and differential controller. Integral controller is not used because the observer used in the control system works in the same way with the controller. In order to reject the disturbance heat flow, VHDOB is used and the model of a Peltier device is changed according to temperature $T_{\mathrm{p}}$ and reference value of the current $I_{\mathrm{a}}^{\mathrm{ref}}$. In the conventional HDOB, the nonlinear elements such as a Joule heat and heat flow inside the Peltier device are not considered. In the next section, some experiments are conducted to verify the proposed method.

\section{Experiments}

5.1 Experimental Setup Figure 9 shows the experimental setup in this paper. The Peltier device that the side is 
Table 1. Parameters of Experiments

\begin{tabular}{c|c|c}
\hline Para. & Description & Value \\
\hline \hline$K_{\mathrm{p}}$ & Proportional gain & $1.0($ Ex. 1) \\
& & $0.5,1.0,1.5($ Ex. 2) \\
$C_{\mathrm{n}}$ & Nominal thermal capacitance & $2.0 \mathrm{~J} / \mathrm{K}$ \\
$R_{\mathrm{pn}}$ & Nominal thermal resistance & $5.0 \mathrm{~W} / \mathrm{K}$ \\
$R_{\mathrm{en}}$ & Nominal electric resistance & $4.1 \Omega$ \\
$\alpha_{\mathrm{n}}$ & Nominal seebeck coefficient & $0.023 \mathrm{~V} / \mathrm{K}$ \\
$g_{\mathrm{d}}$ & Cut-off frequency of HDOB & $1.0 \mathrm{rad} / \mathrm{s}(\mathrm{Ex} .1)$ \\
& & $0.5,1.0,1.5 \mathrm{rad} / \mathrm{s}($ Ex. 2) \\
$g_{\mathrm{p}}$ & Cut-off frequency for pseudo differential & $10.0 \mathrm{rad} / \mathrm{s}$ \\
\hline \hline
\end{tabular}

$20 \mathrm{~mm} \times 20 \mathrm{~mm}$ was attached on a heat sink. Two thermocouples were used for sensing both sides of the Peltier device, and attached to the center of the device using thermal conduction sheet. The Peltier device was controlled to $-5.0 \mathrm{~K}$ (relative temperature). Two kinds of experiments were conducted to show that temperature responses of responsiveness and robustness against a disturbance. In the first experiments, the temperature responses of the proposed method were compared with a normal HDOB and a proportional controller. The second experiments were conducted in case of changing a proportional gain and a cut-off frequency of VHDOB. In the case of changing the cut-off frequency, human finger contacts to the Peltier device after 25 seconds. The values of parameter used for these experiments are shown in Table 1. Here, the thermal capacitance of the Peltier device was derived from some identify tests. In the test, the thermal resistance is calculated from the theoretical value. The electric resistance is measured by using digital multi-meter. The thermal response is oscillatory because of the noise caused by the power supply. Therefore, the cut-off frequency is set to low value such as $1.0 \mathrm{rad} / \mathrm{s}$. The time constants of temperature sensing can be improved by reducing noises from the thermal system especially an AD conversion circuit. The improvement of the system will be conducted in the future work.

5.2 Experimental Results Figure 10 shows the results of temperature control of the Peltier device to $-5 \mathrm{~K}$ (relative temperature) comparing to the case of using normal $\mathrm{P}$ controller, normal HDOB, and VHDOB. In case of normal proportional controller, there is a steady-state error caused by an ambient air and external heat flow. It is found that the thermal system receives large influence on an external disturbance. In comparison with the normal HDOB, time until the steady response of VHDOB was short. This is because a modeling error is reduced by considering the nonlinear element, such as a Joule heat and a heat flow inside the device. Figure 11 shows the temperature responses of the proposed method comparing varied proportional gain. From Fig. 11, the response speed becomes fast as increasing the proportional gain. Figure 12, the temperature responses with contact after 25 seconds comparing varied cut-off frequency of the observer. From Fig. 12, response speed against a disturbance heat flow becomes fast as increasing the cut-off frequency. Therefore, it can be said that the VHDOB effectively works. By taking a nonlinear element of the thermal system into account to the model, a modeling error is reduced, and the control gain or cut-off frequency can be set higher comparing to the normal HDOB. From these experiments, robust temperature control can be conducted by using the proposed method.

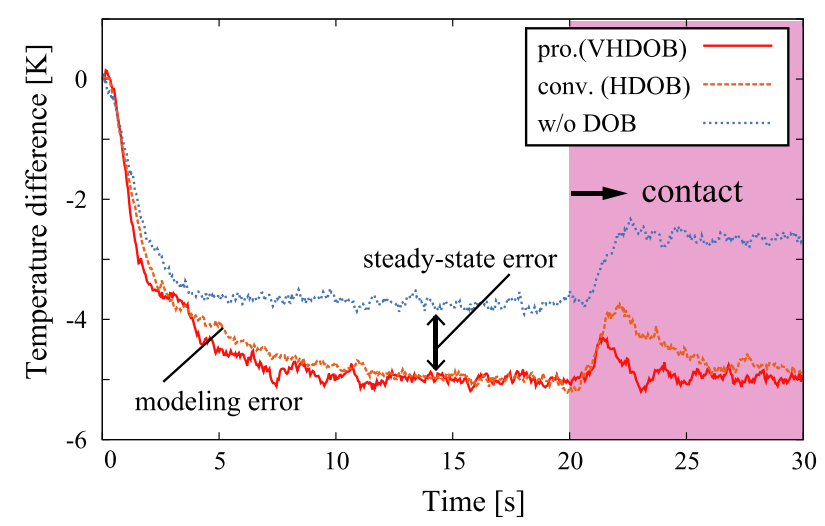

Fig. 10. Experimental results of temperature control comparing conventional method (Ex. 1)

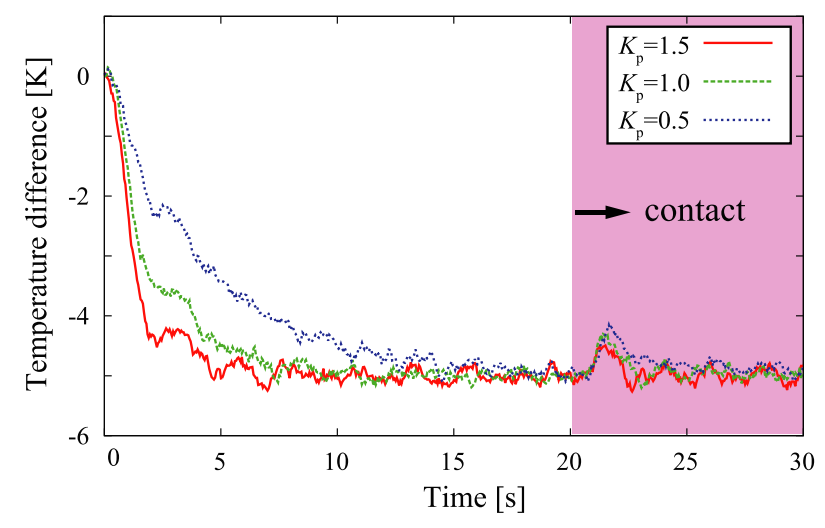

Fig. 11. Experimental results of temperature control with changing proportional gain (Ex. 2)

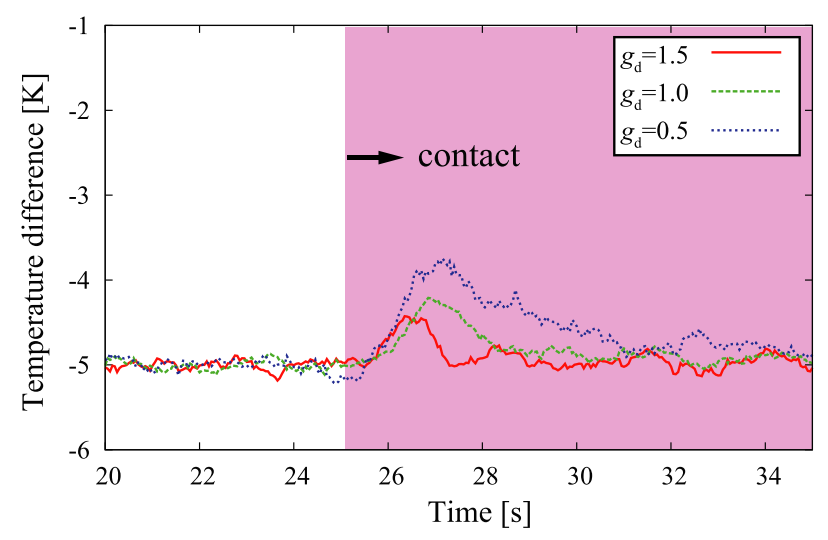

Fig. 12. Experimental results of temperature control with contact (Ex. 2)

\section{Effect of Modeling Error}

In this section, effect of modeling error is discussed. Main claim focuses on an effect of thermal resistance because it is the dominant factor of instability VHDOB. It is analyzed based on a Nyquist diagram using describing function technique. The variable observer is divided into a linear system and a nonlinear system, shown in Fig. 13. Here, $G$ denotes the low pass filter, expressed as

$$
G=\frac{g_{\mathrm{d}}}{s+g_{\mathrm{d}}}
$$

From Fig. 13, the characteristic equation is calculated as 


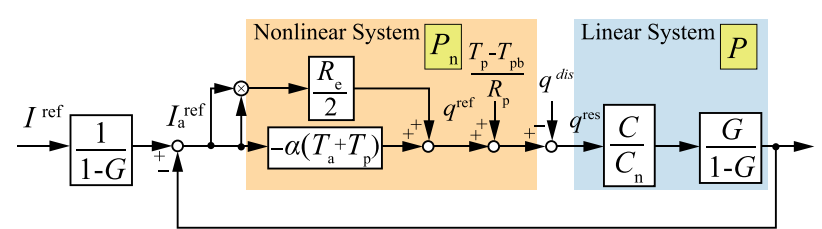

Fig. 13. Equivalent block diagram of VHDOB

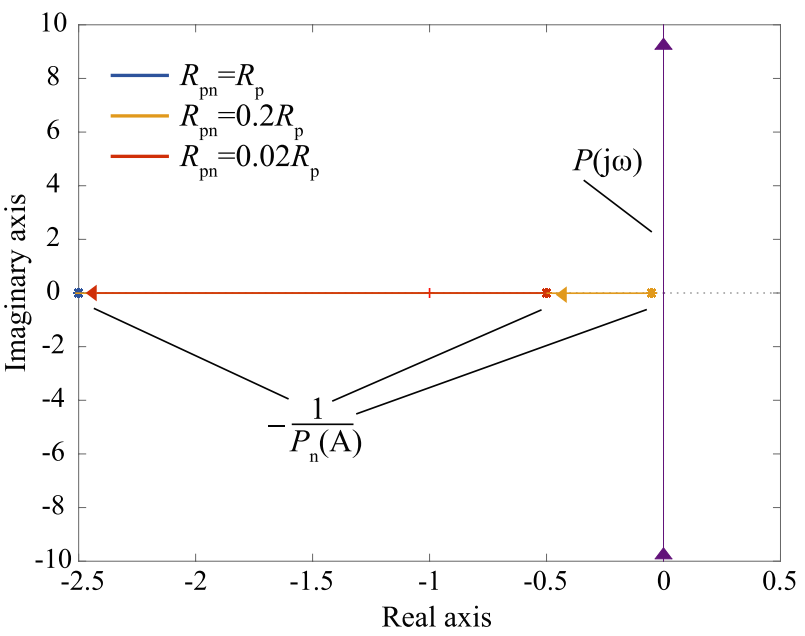

Fig. 14. Nyquist diagram with modeling error

$$
\begin{aligned}
& 1+P_{\mathrm{n}}(A) P(s)=0 \ldots \ldots \ldots \ldots \ldots \ldots \ldots \ldots \ldots \ldots \ldots \ldots \ldots \\
& P_{\mathrm{n}}(A)=-\alpha\left(T_{\mathrm{a}}+T_{\mathrm{p}}\right) A+\frac{R_{\mathrm{e}}}{2} A^{2}+\frac{T_{\mathrm{p}}-T_{\mathrm{pb}}}{R_{\mathrm{p}}} \cdots \\
& P(s)=\frac{C}{C_{\mathrm{n}}} \frac{G}{1-G}, \cdot
\end{aligned}
$$

where $A$ stands for an amplitude of the input signal. The nonlinear elements are effected from the amplitude of the input, and the stability of the observer can be analyzed from the relationship between an amplitude locus of the nonlinear element and a frequency locus of the linear element. The relationship between $P_{\mathrm{n}}$ and $P$ is derived as

$$
-\frac{1}{P_{\mathrm{n}}(A)}=P(s)
$$

The relationship shown in (12) is expressed based on the Nyquist diagram, shown as Fig. 14. Here, the modeling errors $\left(R_{\mathrm{n}}=0.2 R, 0.02 R\right)$ is considered. In the analysis, temperature difference between both sides of a Peltier device sets to 2.0. From Fig. 14, it is found that modeling error of the thermal resistance of a Peltier device $R_{\mathrm{p}}$ has great effect on the stability margin of the system. The range of the modeling errors shown in Fig. 14 is stable; however, the observer becomes unstable in the case of including large modeling error of $R_{\mathrm{p}}$. Therefore, identification of the thermal resistance of a Peltier device is important to use the proposed observer effectively.

\section{Conclusion}

In this paper, the variable heat disturbance observer (VHDOB) considering the nonlinear characteristics of the Peltier device is proposed. The nonlinear elements such as Joule heat and heat flow inside the device are considered in the thermal model used in the proposed method. From the proposed method, the robustness against disturbance can be improved by decreasing the modeling error. Some experiments were conducted to show that temperature responses of responsiveness and robustness against the disturbance. These experimental results showed the validity of the proposed method comparing to the normal proportional controller, normal HDOB. In addition, the stability analysis of the observer was conducted. From the analysis, the modeling error of the thermal resistance has a large effect on the stability of the proposed observer, and it is found that the identification of the thermal resistance is important to use the proposed observer effectively. This method is expected to extend a field that the device can be used.

\section{Acknowledgment}

This work was partially supported by JSPS KAKENHI $18 \mathrm{H} 03784$.

\section{References}

( 1 ) G.J. Monkman and P.M. Taylor: "Thermal Tactile Sensing", IEEE Trans. RA, Vol.9, No.3, pp.313-318 (1993)

( 2 ) M.B. Khoudja, M. Hafez, J.M. Alexandre, J. Benachour, and A. Kheddar: "Thermal Feedback Model for Virtual Reality", Proc. IEEE MHS, pp.153158 (2003)

( 3 ) A. Yamamoto, B. Cros, H. Hashimoto, and T. Higuchi: "Thermal Tactile Presentation with On-Site Parameter Identification of Finger", Proc. IEEE ISIE, pp.1365-1370 (2005)

( 4 ) S. Ino, S. Shimizu, T. Odagawa, M. Sato, M. Takahashi, T. Izumi, and T. Ifukube: "A Tactile Display for Presenting Quality of Materials by Changing the Temperature of Skin Surface", Proc. IEEE RHC, pp.220-224 (1993)

( 5 ) H. Morimitsu and S. Katsura: "Two-Degree-of-Freedom Robust Temperature Control of Peltier Device Based on Heat Disturbance Observer", IEEJ Trans. IE, Vol.13, No.7, pp.967-973 (2011)

( 6 ) M. Guitani, A. Benallegue, and A. Kheddar: "Learning-Based Thermal Rendering in Telepresence", Lecture Notes in Computer Science, Vol.5024, pp.820-825 (2008)

( 7 ) H. Morimitsu and S. Katsura: "Sensorless Control of Heat Inflow to a Thermal Display Using a Heat Inflow Observer", IEEE Trans. Ind. Electron., Vol.62, No.7, pp.4288-4297 (2015)

( 8 ) A. Drif, J. Citérin, and A. Kheddar: "Thermal Bilateral Coupling in Teleoperators”, Proc. IEEE/RSJ IROS, pp.1301-1306 (2005)

( 9 ) M. Guitani and A. Kheddar: "Modeling Identification and Control of Peltier Thermoelectic Modules for Telepresence", Journal of Dynamic Systems, Measurement, and Control, Vol.133, No.3, pp.1-8 (2011)

(10) Y. Osawa, H. Morimitsu, and S. Katsura: "Control of Thermal Conductance with Detection of Single Contacting Part for Rendering Thermal Sensation", IEEJ Journal of Industry Applications, Vol.5, No.2, pp.101-107 (2016)

(11) S. Gallo, L. Santos-Carreras, G. Rognini, M. Hara, A. Yamamoto, and T. Higuchi: "Towards Multimodal Haptics for Teleoperation: Design of a Tactile Thermal Display", Proc. IEEE AMC, pp.1-5 (2012)

(12) F. Felgner, L. Exel, M. Nesarajah, and G. Frey: "Component-Oriented Modeling of Thermoelectric Devices for Energy System Design", IEEE Trans. Ind. Electron., Vol.61, No.3, pp.1301-1310 (2014)

(13) I. Darian-Smith and K.O. Johnson: "Thermal Sensibility and Thermoreceptors", Journal of Investigative Dermatology, Vol.69, No.1, pp.146-153 (1977)

(14) A. Singhal and L.A. Jones: "Space-Time Interaction and the Perceived Location of Cold Stimuli", Proc. IEEE HAPTICS, pp.92-97 (2016)

(15) A. Manasrah, N. Crane, R. Guldiken, and K.B. Reed: "Perceived Cooling Using Asymmetrically-Applied Hot and Cold Stimuli”, IEEE Trans. HAPTICS, Vol.10, No.1, pp.75-83 (2017)

(16) S.L. Macknik and S. Martinez-Conde: "No Brain No Pain”, Journal of Scientific American Mind, Vol.24, pp.19-21 (2013)

(17) R. Watanabe, R. Okazaki, and H. Kajimoto "Mutual Referral of Thermal Sensation Between Two Thermal-Tactile Stimuli", Proc. IEEE HAPTICS, pp.299-302 (2014)

(18) W. Gao, S. Emaminejad, H.Y. Nyein, S. Challa, K. Chen, A. Peck, H.M. Fahad, H. Ota, H. Shiraki, D. Kiriya, D. Lien, G.A. Brooks, R.W. Davis, and A. Javey: "Fully Integrated Wearable Sensor Arrays for Multiplexed in Situ Perspiration Analysis", Nature, Vol.529, pp.509-514 (2016)

(19) W. Honda, S. Harada, T. Arie, S. Akita, and K. Takei: "Printed Wearable 
Temperature Sensor for Health Monitoring", Proc. IEEE Sens., pp.22272229 (2014)

(20) A. Uchiyama, T. Hamatani, and T. Higashino: "Estimation of Core Temperature based on a Human Thermal Model Using a Wearable Sensor", Proc. IEEE GCCE, pp.605-609 (2015)

(21) T. Yokota, Y. Inoue, Y. Terakawa, J. Reeder, M. Kaltenbrunner, T. Wared, K. Yange, K. Mabuchif, T. Murakawag, M. Sekino, W. Voitc, T. Sekitani, and T. Someya: "Ultraflexible, Large-Area, Physiological Temperature Sensors for Multipoint Measurements", Proc. NAS, Vol.112, No.47, pp.14533-14538 (2015)

(22) M. Bartkowiak and G.D. Mahan: "Heat and Electricity Transport through Interfaces", Semiconductors and Semimetals, Vol.70, pp.245-271 (2001)

(23) K.H. Lee and O.J. Kim: "Analysis on the Cooling Performance of the Thermoelectric Micro-Cooler", International Journal of Heat and Mass Transfer, Vol.50, pp.1982-1992 (2007)

(24) L.W. da Silva and M. Kaviany: "Micro-Thermoelectric Cooler: Interfacial Effect on Thermal and Electrical Transport", International Journal of Heat and Mass Transfer, Vol.47, pp.2417-2435 (2004)

(25) W. Sato and N. Isoshima: "Thermal Network Model-Based Data Assimilation for Estimation of Temperature Characteristic Coefficients for Peltier Device", Trans. JSME, Vol.82, No.844, pp.207-220 (2016) (in Japanese)

(26) H.Y. Zhang: "A General Approach in Evaluating and Optimizing Thermoelectric Coolers", Journal of Cryogenics, Vol.33, No.6, pp.1187-1196 (2010)

(27) X.C. Xuan, K.C. Ng, C. Yap, and H.T. Chua: "The Maximum Temperature Difference and Polar Characteristic of Two-Stage Thermoelectric Coolers", International Journal of Refrigeration, Vol.42, No.5, pp.273-278 (2002)

(28) A. Sarikhani and O.A. Mohammed: "Inter-Turn Fault Detection in PM Synchronous Machines by Physics-Based Back Electromotive Force Estimation", IEEE Trans. Ind. Electron., Vol.60, No.8, pp.3472-3484 (2013)

(29) A.A. Hasmasan, C. Busca, R. Teodorescu, L. Helle, and F. Blaabjerg: "Electro-Thermo-Mechanical Analysis of High-Power Press-Pack Insulated Gate Bipolar Transistors under Various Mechanical Clamping Conditions", IEEJ Journal of Industry Applications, Vol.3, No.3, pp.192-197 (2014)

(30) K. Ohnishi, M. Shibata, and T. Murakami: "Motion Control for Advanced Mechatronics", IEEE/ASME Trans. Mec., Vol.1, No.1, pp.56-67 (1996)
Yukiko Osawa (Member) received her B.E. degree in system design

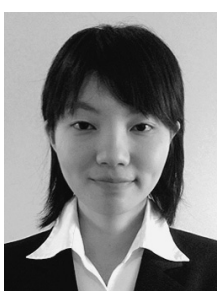
engineering and the M.E. degree in integrated design engineering from Keio University, Yokohama, Japan, in 2015 and 2016, respectively. Since 2016, she has been a Doctor's course student at Keio University, Yokohama, Japan. From 2017, she is a Research Fellow of the Japan Society for the Promotion of Science (JSPS). Her research interests include thermotics, distributed parameter systems, human support space, and human interface. She is a Member of IEEJ, as well as IEEE. She was the recipient of the IEEJ Distinguished Paper Award in 2017.

Seiichiro Katsura (Senior Member) received the B.E. degree in sys-

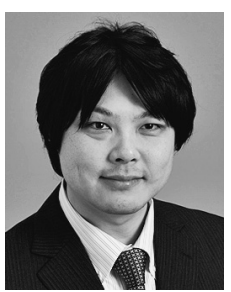
tem design engineering and the M.E. and Ph.D. degrees in integrated design engineering from Keio University, Yokohama, Japan, in 2001, 2002 and 2004, respectively. From 2003 to 2005, he was a Research Fellow of the Japan Society for the Promotion of Science (JSPS). From 2005 to 2008, he worked at Nagaoka University of Technology, Nagaoka, Niigata, Japan. Since 2008, he has been at Keio University, Yokohama, Japan. In 2017, he was a Visiting Researcher with the Laboratory for Machine Tools and Production Engineering (WZL) of RWTH Aachen University, Aachen, Germany. His research interests include applied abstraction, human support, data robotics, wave system, systems energy conversion, and electromechanical integration systems. Prof. Katsura serves as an Associate Editor of the IEEE Transactions on Industrial Electronics. He was the recipient of the IEEJ Distinguished Paper Awards in 2003 and 2017, IEEE Industrial Electronics Society Best Conference Paper Award in 2012, and JSPS Prize in 2016. 